\title{
Toggle-Swing Dispensing Plastic Container Closure
}

National Cancer Institute

\section{Source}

National Cancer Institute. Toggle-Swing Dispensing Plastic Container Closure. NCI

Thesaurus. Code C96135.

A closure with a lower part attaches securely and seals the container. The upper part provides a second movable portion which functions in a rocker-like pivotal motion between an open and a closed position. 